Surgery Center Opioid Use 2018-19

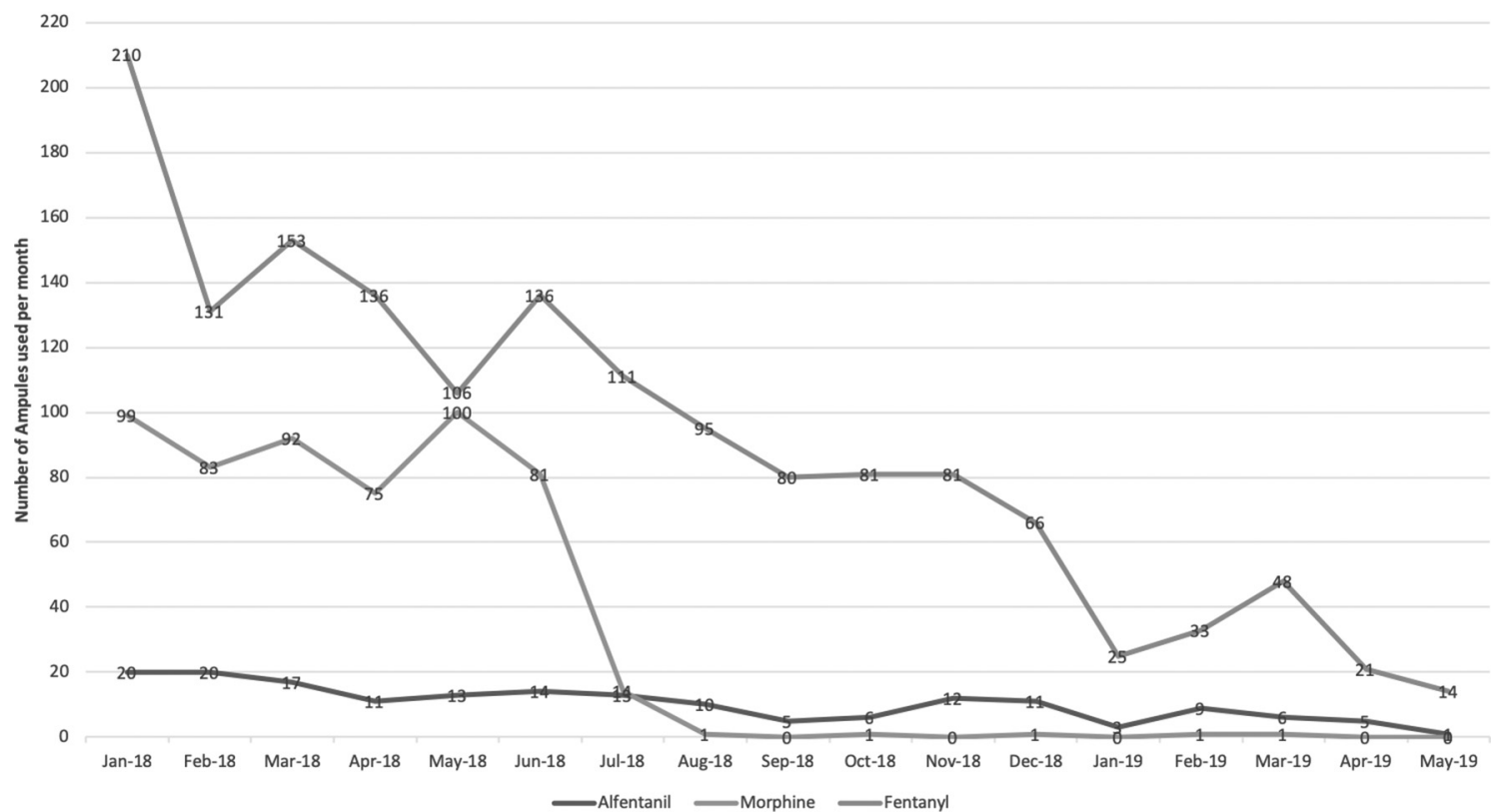

Abstract 12 Figure 1 Monthly vials of opioids used at surgery center

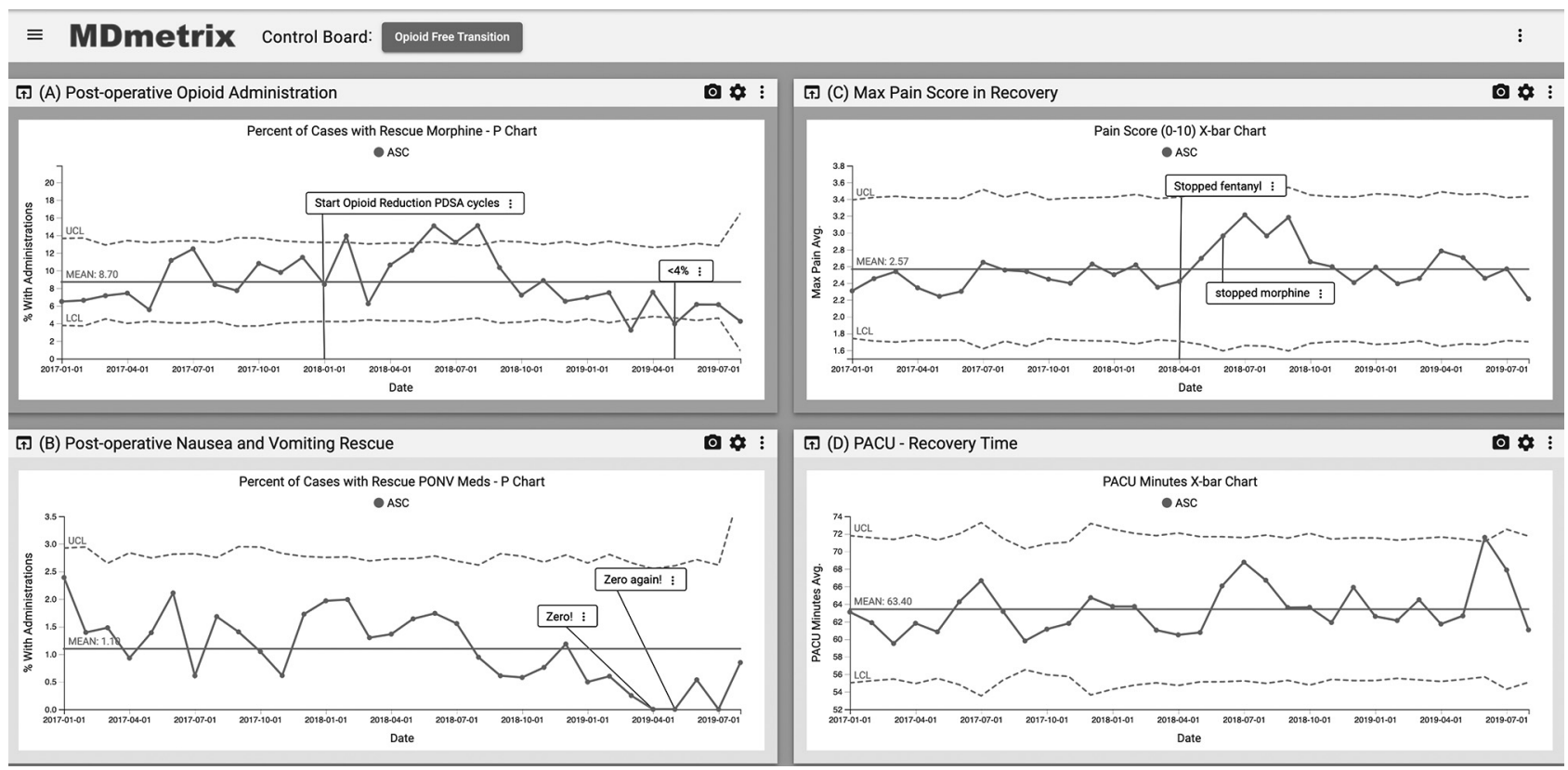

Abstract 12 Figure 2 Balancing measures to track effectiveness of opioid reduction at surgery center.

\section{3 A NOVEL EVIDENCE-BASED APPROACH TO DIGITAL
OUTREACH IMPROVES PATIENT ENGAGEMENT AND
HEALTH OUTCOMES IN TWO DISTINCT COHORTS OF
MEDICAID PATIENTS}

Yolande Pengetnze, Donna Persaud, Albert Karam, Xiao Wang. PCCI, USA

10.1136/bmjoq-2019-ihi.13

Background Patient education and motivation are crucial to behavior change, care engagement, and successful outcomes for chronic conditions such as asthma or term-limited conditions such as pregnancy. Educational programs, if not personalized, might lead to no or short-lived behavior change and suboptimal outcomes.

Objectives Using the framework of education as a continuous, repetitive, and interactive process, and leveraging ubiquitous access to mobile technology, we designed a novel digital outreach program that uses short, focused, motivational text messages targeted and tailored to patients' risk profiles to produce sustained healthy behavior change. Messaging content and frequency are personalized for optimal and sustained effectiveness, while mitigating 'Digital Fatigue' risk. Advanced analytics for accurate predictive patient risk profiling drive patient engagement personalization. 
Methods A quasi-experimental study of the clinical effectiveness of a novel risk-driven digital outreach program in two distinct Medicaid cohorts: Children with asthma and Pregnant women at risk for preterm birth vs. matched controls.

Results Among Medicaid children with asthma, program participation is associated with $24 \%$ increase in outpatient visits, $24 \%$ reductions in emergency department visits, and $15 \%$ increase in controller medication use. Among Medicaid pregnant women, program participation is associated with $24 \%$ increase in prenatal visit attendance, $27 \%$ reductions in early preterm deliveries ( $<35$ weeks), and 54\% reductions in baby costs in the first year of life. Overall Program Satisfaction is high.

Conclusions Targeted text messaging tailored to patient risk profiles drives healthy behavior changes and improved health

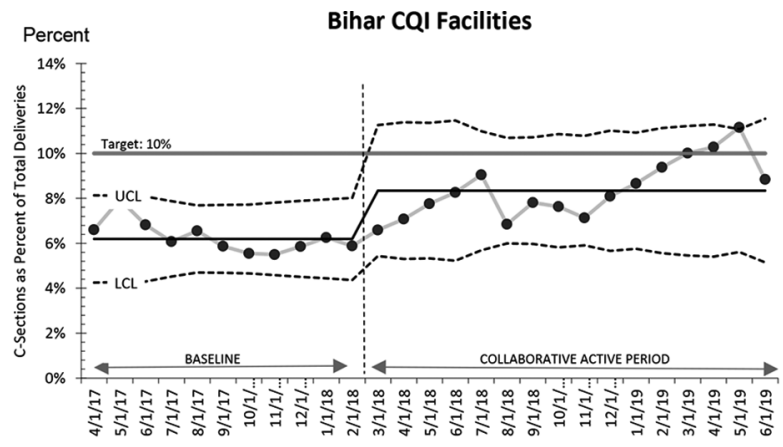

Abstract 14 Figure 1 Prevalence of C-section deliveries

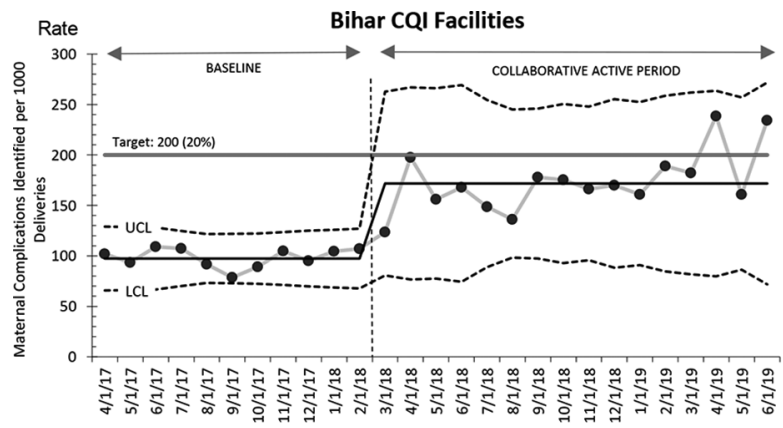

Abstract 14 Figure 2 Rate of identification of maternal complications

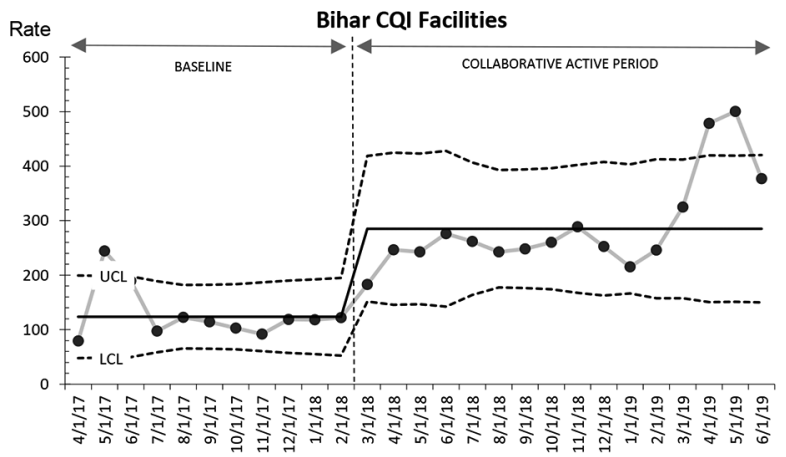

outcomes in diverse health cohorts. Leveraging advanced analytics to drive personalized digital health engagement is essential for success.

\section{A QUALITY IMPROVEMENT COLLABORATIVE TO IMPROVE MATERNAL COMPLICATION IDENTIFICATION AND USE OF C-SECTION AND BLOOD TRANSFUSION IN BIHAR, INDIA}

${ }^{1}$ Clark Jackson, ${ }^{1}$ Abha Mehndiratta, ${ }^{1}$ Gareth Parry, ${ }^{2}$ Hemant Shah, ${ }^{2}$ Tanmay Mahapatra, 2Prabir Moharana, 'Pierre Barker, 'Azhar Ali. ${ }^{1} / H I$, USA; ${ }^{2}$ CARE India, India

10.1136/bmjoq-2019-ihi. 14
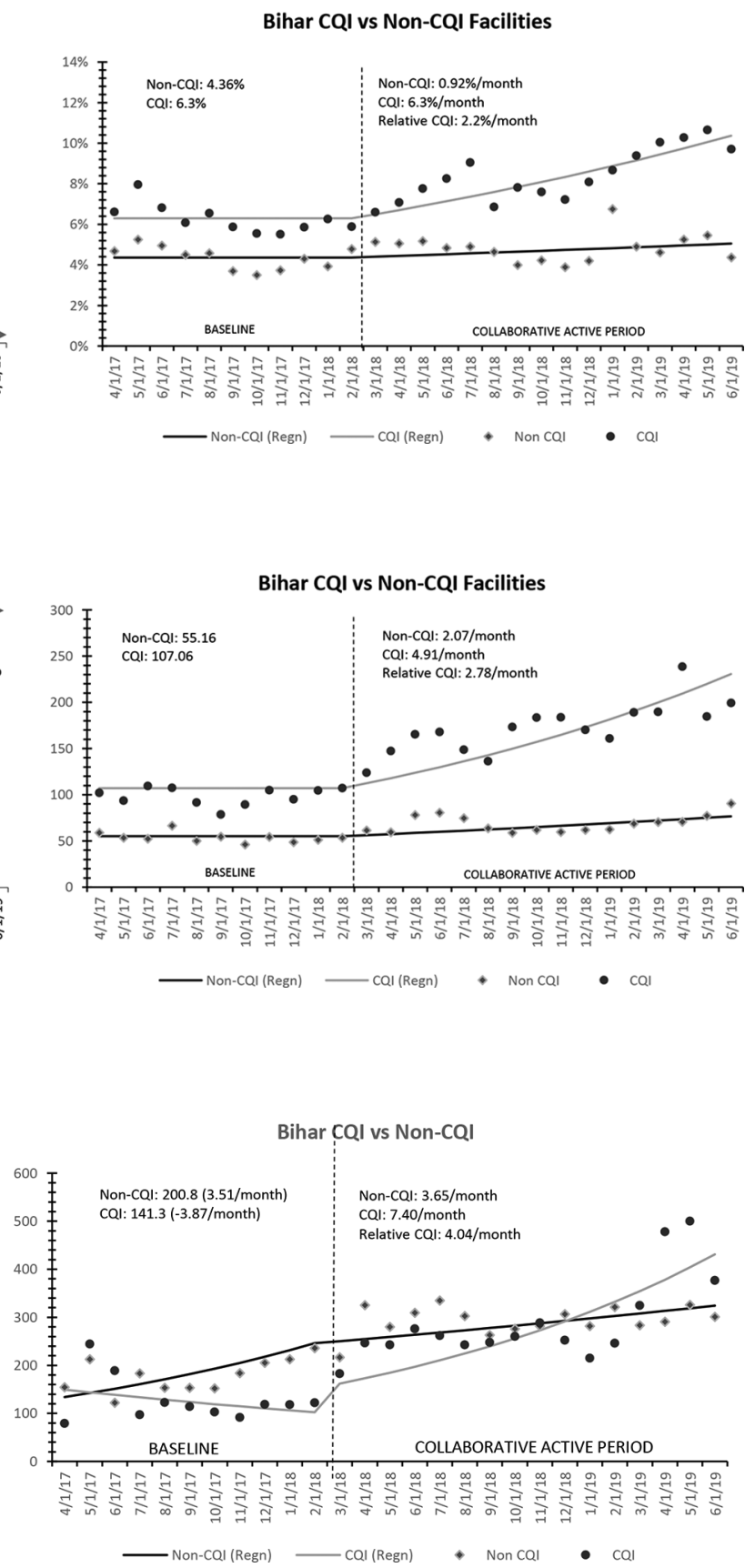

Abstract 14 Figure 3 Rate of complicated pregnancies treated with blood transfusion (per 10,000 institutional deliveries) 